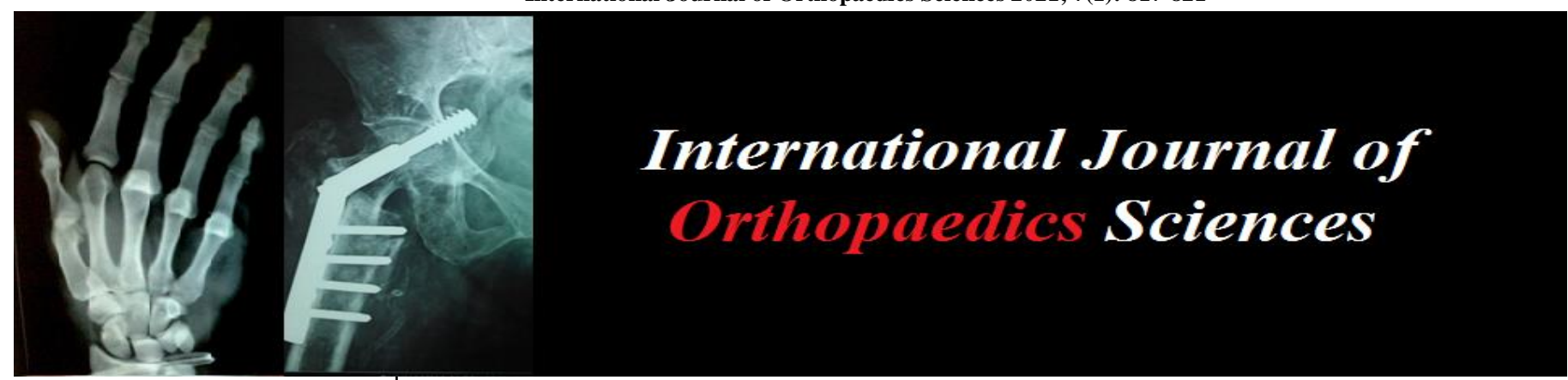

E-ISSN: 2395-1958

P-ISSN: 2706-6630

IJOS 2021; 7(1): 817-821

(C) 2021 IJOS

www.orthopaper.com

Received: 16-11-2020

Accepted: 22-12-2020

Anil Bulagond

Department of Orthopaedics,

Shri B M PATIL medical college

Vijayapur, Karnataka, India

Puneet Chamkeri

Department of Orthopaedics,

JNMC medical college Belagavi,

Karnataka, India

Gireesh Khodnapur

Department of Orthopaedics,

Shri B M PATIL medical college

Vijayapur, Karnataka, India.

Adhitiyaa Elango

Department of Orthopaedics,

Shri B M PATIL medical college

Vijayapur, Karnataka, India

Corresponding Author:

Puneet Chamkeri

Department of Orthopaedics,

JNMC medical college Belagavi,

Karnataka, India

\section{Surgical management of open tibial fracture (type i, ii $\&$ iii) with limb reconstruction system}

\author{
Anil Bulagond, Puneet Chamkeri, Gireesh Khodnapur and Adhitiyaa \\ Elango
}

DOI: https://doi.org/10.22271/ortho.2021.v7.i1m.2574

\section{Abstract}

Background: Tibial diaphyseal fractures are one of the commonest long bone fractures encountered by most of the orthopaedic surgeons. Because most of the length of tibia is subcutaneous throughout, open fractures are more common in tibia than in any other major long bone. Because of high prevalence of complications associated with these fractures, management is often is difficult and the optimum method of treatment remains a subject of controversy. The aim of this study is to evaluate the efficiency of LRS in treatment of open fractures of tibia with other studies in terms of time required for union, rate of malunion and malrotation, rate of infection and range of motion of ankle and knee joint.

Materials and Methods: This study was performed with 25 open fractures of tibia with LRS. All the cases were fresh fractures and traumatic in nature. They were done with procedure as early as possible and the secondary procedures of skin grafting and musculocutaneous flap were done when needed. The cases were followed up for an average period of 6-24 months.

Results: Open fractures of the tibial shaft managed with LRS gave good functional results and patient satisfaction. It involves minimal surgical trauma and less blood loss. It provides the advantages of early ambulation, lower rates of infection, delayed union, nonunion and malunion compared to other treatment modalities. It provides with early weight bearing and earlier returns to work.

Conclusions: It was concluded that early stabilization of open tibial fracture with LRS with immediate soft tissue coverage resulted in good fracture union and minimal rates of complications compared to other modalities of treatment. It is cost effective with minimal hospital stay and early return to work. Over all morbidity is reduced and better patient satisfaction noted.

Keywords: Limb reconstruction system, open tibial fracture

\section{Introduction}

Injuries and fractures have become so common in the present day, main reason being increased population, with increase in vehicular traffic congestion and urbanization, mechanization and agriculturization. The tibia being the most commonly fractured long bone ${ }^{1}$ and its fracture management contributes significantly to the cost of orthopaedic care being provided worldwide. Epidemiological studies suggest that motor vehicle accidents are the most common causes of tibial diaphyseal fractures, followed by sports related injuries. High energy trauma which imparts more kinetic energy that causes fractures which are often severe with associated soft tissue injury.

Treatment options for tibial fractures vary according to the type of fracture, age group, bone density, soft tissue status and associated complications. Conservative methods used are casting or bracing for stable closed fractures. Because of improper anatomical alignment and associated soft tissue injuries, these conservative methods have become less useful.

Operative techniques used are fixation with plates and screws, intramedullary nailing and external fixation. Intramedullary interlocking nails used widely for fixation of unstable, closed and type I and type II compound tibial fractures. External fixators are indicated for type IIIA, IIIB and IIIC compound fractures, severely contaminated fractures, delayed treatment of open tibial fractures, open periarticular fractures and in polytraumatized patients. ${ }^{[1]}$

By its very location, the tibia is exposed to frequent injury ${ }^{1}$. Because one-third of the tibial surface is subcutaneous, through most of its length, open fractures are more common in the 
tibia than in any other major long bone. Further the blood supply to the tibia is more precarious than that of bones enclosed by heavy muscles especially in the lower third which is covered by only tendons. ${ }^{[1]}$

The management of compound fractures of tibia is an enigma to orthopedic surgeon. Numerous methods have been described and came out with varying results for the management of compound fractures of tibia. Introduction of external fixator is a revolution in the management of compound fractures of tibia and it has saved many limbs from amputation. In external fixation; fracture fragments can be realigned, compressed or distracted, without the need of opening fracture site.

With improved components, and a better understanding of the principles, that govern their safe and effective use, external fixator have become indispensable tools in the hands of the experienced trauma surgeon. ${ }^{2}$ External fixators have the unique capability to stabilize bone and soft tissues at a distance from the operative or injury focus. ${ }^{2} \mathrm{~A}$ planned approach is necessary before using any external fixator. Appropriate roentgenogram is essential. A complete set of instruments should be available.

In our study, we have used unilateral frame with half pins, the system is named as Limb Reconstructive System (LRS). This system is very effective, and offers rigid stabilization of fracture fragments and with an access to soft tissue care. Though, initially these fixators are expensive, but on a long run they are cheap, as these can be repeatedly used without any compromise. Our patients are from the rural side, and the use of such a simple and effective external fixator is necessary in order to reduce the economic burden and to obtain the maximum benefit. This study analyzes the efficacy of LRS external fixation in achieving anatomical reduction, stable fixation, early return to function, results with respect to bony union, soft tissue coverage and with special consideration of knee and ankle movements, limb shortening and disadvantages.

\section{Materials and Methods}

25 cases of open fractures of both bones of leg were selected, treated and followed up between November 2010 and August 2012 at Mysore medical college and research institute, Mysore, Karnataka. Both male and female patients were included under the study, most common causes were of road traffic accidents. Maximum number of patients were between 20 and 40 years. Thorough examination was done to rule out other systemic injury like head injury, cardiorespiratory and abdominal status. Patients with hypovolaemic shock were treated with IV fluids like plasma expanders, dextrose, normal saline, ringer lactate solution. Immediate intravenous antibiotics, intramuscular tetanus toxoid and tetanus immunoglobulin were given. Mean while airway and breathing was maintained. If needed, according to severity of soft tissue damage and degree of contamination, patients were given anti gas gangrene serum, 5,000-10,000 IU infusion in dextrose after test dose within 24 hours.

Once the patient is haemodynamically stabilized, clinical evaluation and primary wound debridement was done in OT under anaesthesia. Wounds were graded according to Gustilo Anderson's classification as Type 1, 2, 3A, 3B and 3C based on the size of wound, degree of soft tissue injury, level of contamination, degree of bony injury and presence or absence of neurovascular injury. In our study type II and type IIIA and type IIIB were common. Wound debridement was done under spinal anaesthesia, 5 to 6 litre of normal saline, betadine and hydrogen peroxide were used. Antiseptic solution irrigation for washing wound is used. For type II and type IIIA and IIIB fractures, thorough wound debridement was done and shifted to radiology department for X-ray evaluation. In our series, compound lower one-third fracture both bones of leg were more common. Slightly common on right side than the left leg. In our series, segmental fractures were seen in 2 cases and comminuted compound fracture in 4 cases. Butterfly fragment in 5 cases and oblique fracture in 3 cases and transverse fracture in 11 cases. Application of LRS external fixator was carried out in major OT after investigations.

Under spinal anaesthesia and with tourniquet in situ, thorough debridement of wound was performed which was followed by LRS application. Stab incisions were followed by blunt dissection until the bone was reached. Shantz pins were passed proximal and distal to fracture site followed by insertion of rail rods and clamps. In simple fractures without bone loss, LRS was applied in acute docking mode and immediate weight bearing was advised. In those with bone loss, compression distraction technique was used at $1 \mathrm{~mm}$ for every week alternatively in immediate postoperative period. When required, skin grafting and flap surgeries were carried out within 3-4 weeks. Patients were asked to come for followup at every 4 weeks interval and weekly dressings were advised to prevent pin tract infection. Clinical and radiological assessment was done at every follow-up to check for pin tract infection and presence of callus.

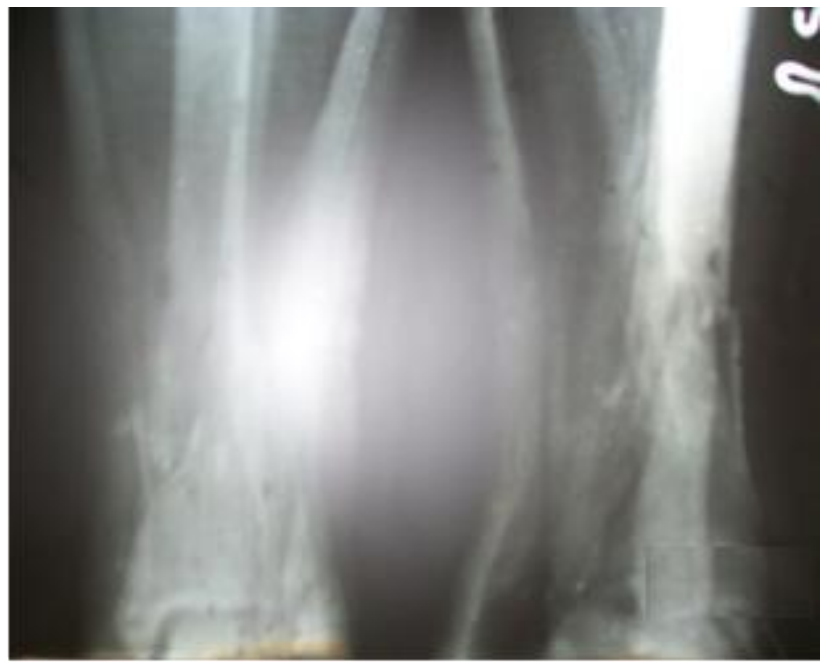

Fig 1: Pre-op X-ray of tibia



Fig 2: At 1 month follow-up 


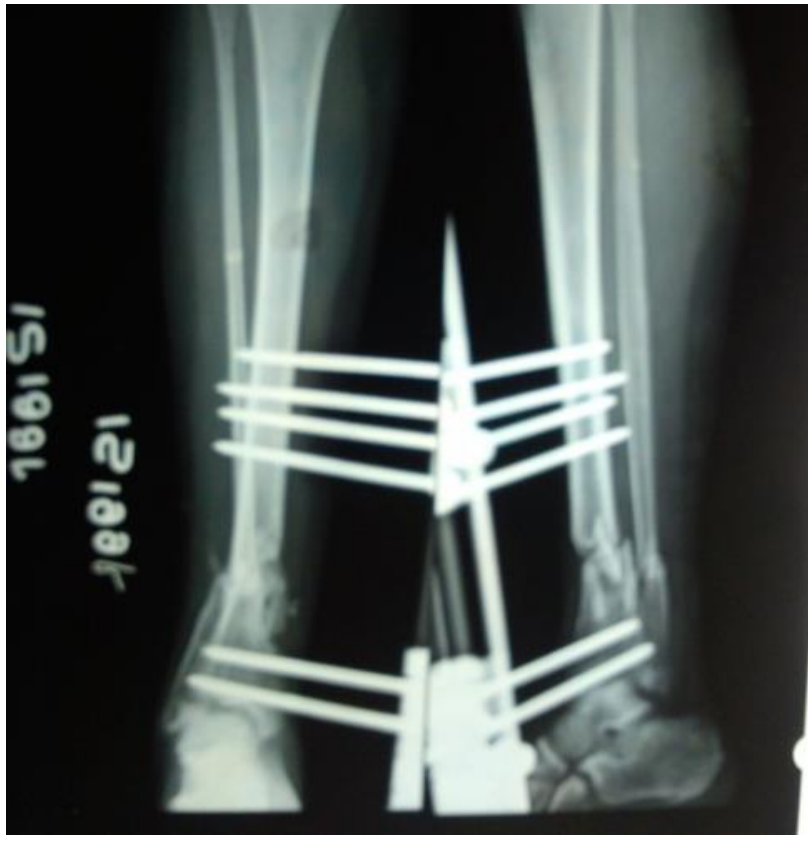

Fig 3: At 4 month follow-up

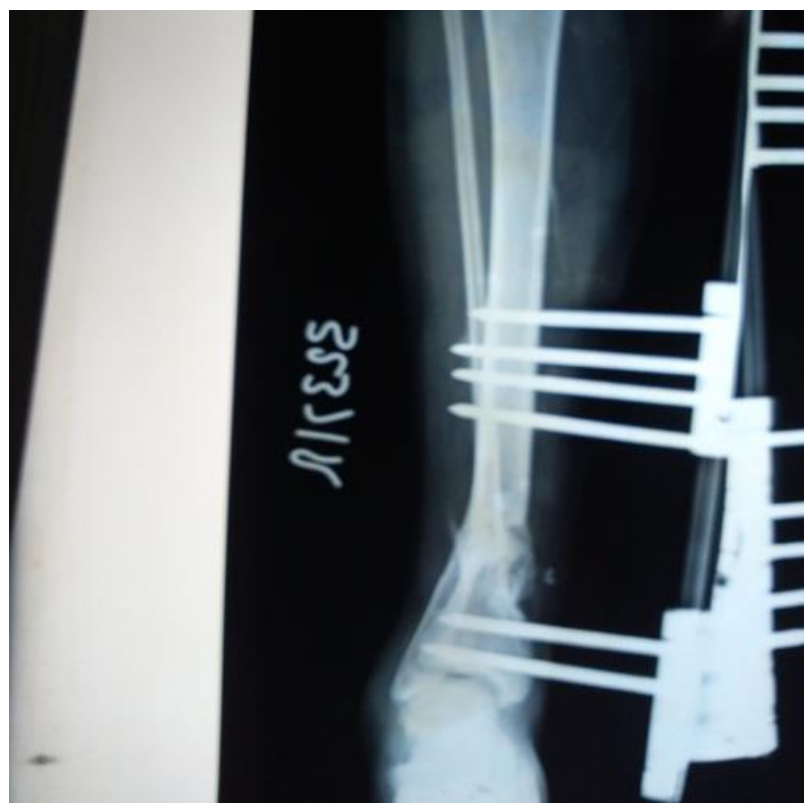

Fig 4: At 8 month follow-up



Fig 5: Knee extension and dorsiflexion after LRS removed

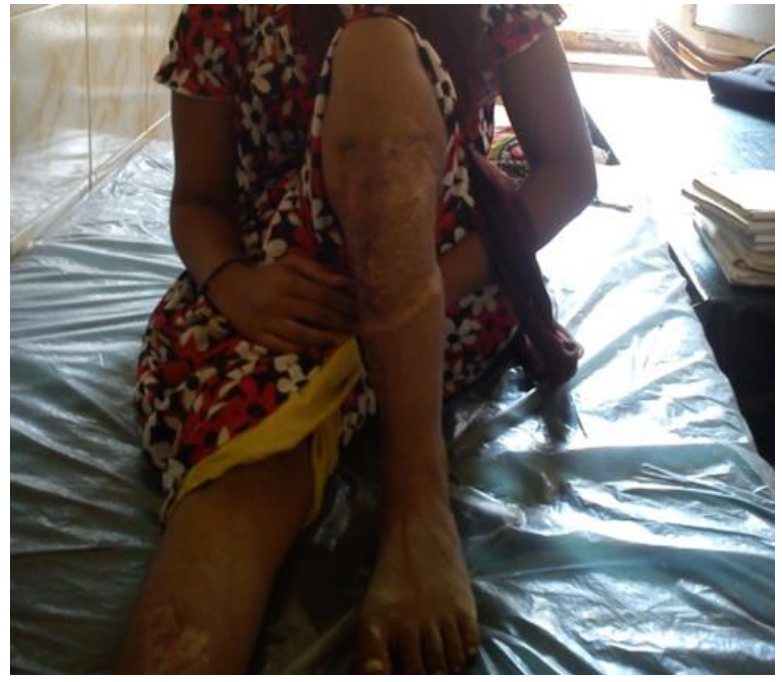

Fig 6: Knee flexion after LRS removed

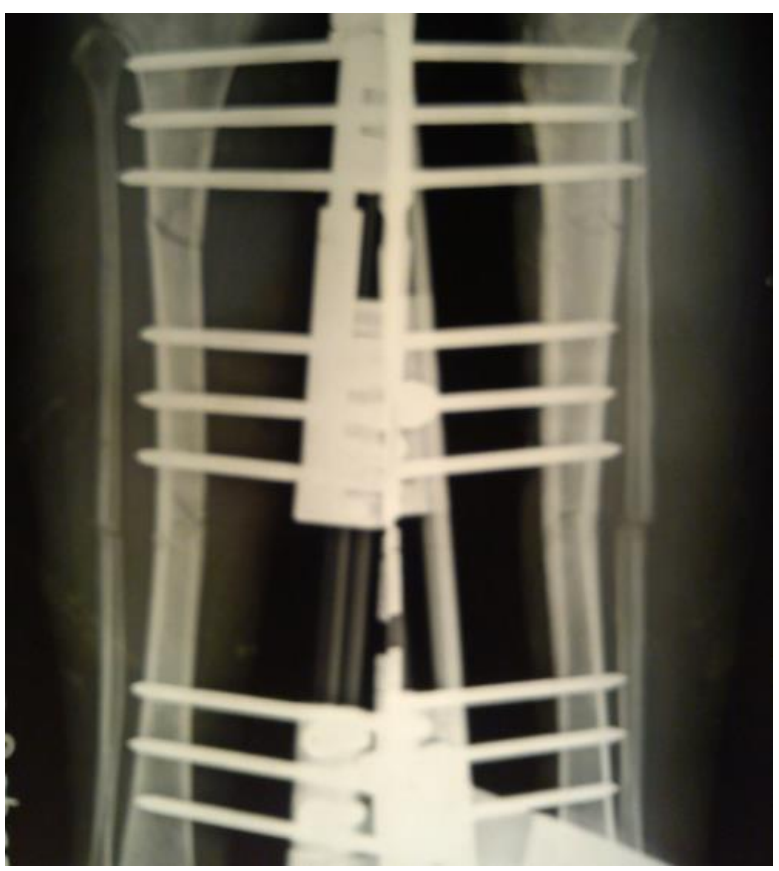

Fig 7: Immediate postoperative X-ray showing segmental fracture of tibia stabilised with LRS fixator

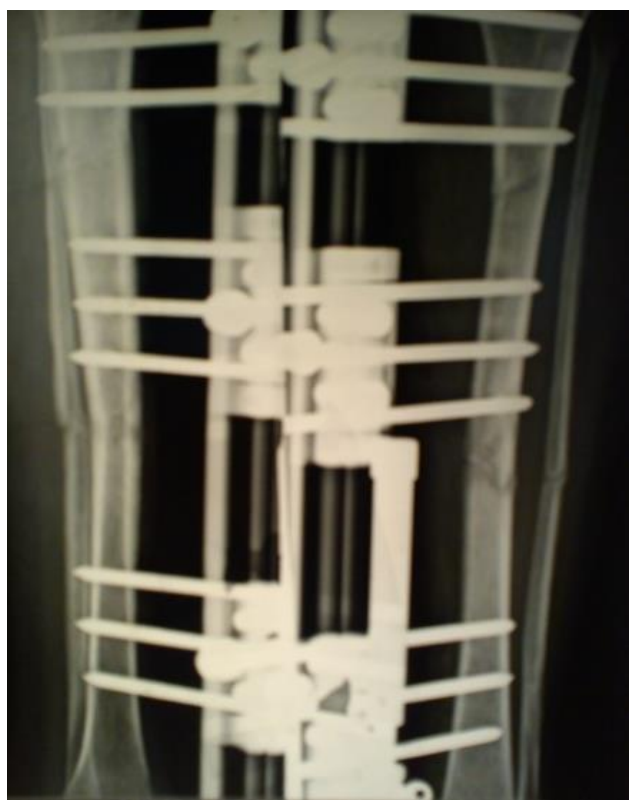

Fig 8: At 6 month follow-up X-ray 


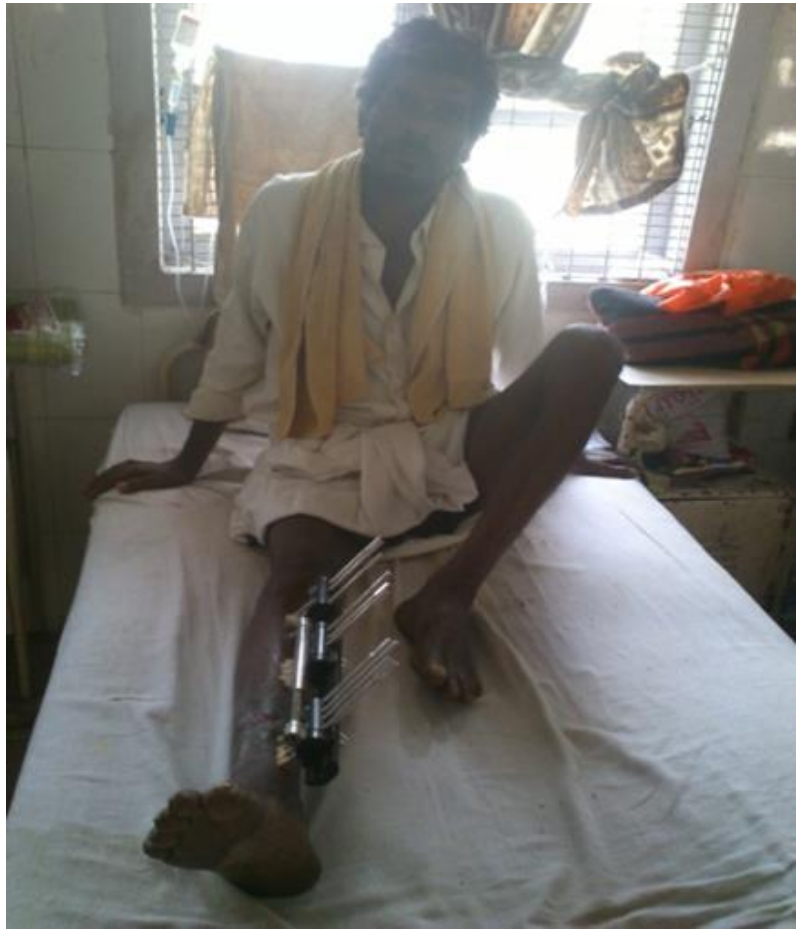

Fig 9: Post-operative X-ray

\section{Results}

In the present study 13 patients were in the mean age of 37 years. $72 \%$ of the patients were male, which may suggest higher level of activities and mobility among males than females. The road traffic accidents were the cause of injury in all cases. Road traffic accident was the cause of injury in $81.9 \%$ of patients in group A and $90 \%$ of patients in group B. In the present study maximum number of patients were belonging to Type II Gustilo-Anderson's group accounting for $52 \%$, followed by Type IIIA and Type IIIB group accounting for $24 \%$ of patients each. In our series of study, $48 \%$ of the fractures were in lower third, $28 \%$ fractures at middle third and $16 \%$ fractures were at proximal third and the remaining $8 \%$ fractures were segmental.

8 patients underwent split skin grafting (32\%), one patient underwent cross leg flap, one more patient underwent muscle pedicle flap and delayed primary closer was done in two patient and secondary closer was done in one patient. A free vascular flap in 5 patients and a local flap repair in 4 patients corrected the soft tissue loss. All soft tissue transfers were successful, except in 1 case, which was infected and treated with antibiotics, healed later. One case of type IIIA comminuted showed signs of non-union on radiology required bone grafting was done. One patient required pin replacement. The most common complications were pin tract infections and shortening of limb occurred in 7 patients each $(28 \%)$. We treated these pin tract infections with suitable parenteral antibiotics after culture and sensitivity and except one, all six cases responded well. In the present study, good results were obtained in 18 patients $(72 \%)$, moderate in 4 patients $(16 \%)$ and poor in 3 patients $(12 \%)$.

\section{Discussion}

Open fractures of tibia are very common in this modern world because of high velocity road traffic accidents. Although newer and better treatment approaches for the management of open fractures are available, open fractures remain to be one of the important challenges in orthopaedic trauma. Because the study has been done in a tertiary care centre, a lot of compound fractures were encountered. According to Behrens and Searls ${ }^{[2]}$, every year two cases out of 1000 injuries were compound tibial fractures and this rate was even greater than $0.2 \%$ in developing countries. The various modalities of treatment available for tibial compound fractures are minimal osteosynthesis, biological fixation and internal fixation with intramedullary nailing or external fixation with different types of fixators. Irrespective of which treatment method is used, the aim of the surgery should be to obtain maximum functionality to the fractured extremity and to maintain patient's life quality with minimum damage or complication [3-5].

Use of external fixators in comminuted, defective, and contaminated open fractures like Gustilo-Anderson types IIIB and IIIC open fractures, is routinely accepted these days ${ }^{[6,7]}$. According to Yokoyama K, treatment of grade IIIB and IIIC with intramedullary nailing was risky as it lead to deep infection and nonunion in $20.3 \%$ cases ${ }^{[8]}$. Therefore, external fixators are preferred modality because they are easy to use and allow soft tissue treatment. But the problems associated are prolonged immobilization and need for revision surgery for definitive fixation at a later stage. Therefore, LRS, which is different from the simple external fixators in allowing full weight bearing immediate postoperatively like an intramedullary fixation was used. LRS fixation technique also has an added advantage of salvaging the limb and preventing amputation. On other side, it has its own complications like pin loosening and pin tract infection. In this case series of 54 cases, pin loosening was noted and pin replacement was required only in $1.85 \%$ cases and moreover, in $92.59 \%$ cases, complete union was achieved within 8 months. The complications of nailing or fixators with acute docking are shortening, soft tissue healing problems, increased morbidity, multiple surgeries, prolonged hospital stay and its consequences like deep vein thrombosis, bedsores, nosocomial infection which eventually leads to increased chances of mal union and non-union and increased financial burden ${ }^{[9]}$. In a study conducted by Edward in 1988, Grade III open tibial fractures were treated with external fixator, where in $93 \%$ of the fractures united well and $89 \%$ patients had satisfactory clinical function ${ }^{16}$. Even in patients treated with secondary nailing after primary external fixator or after delayed primary nailing there are more chances of infection ${ }^{10}$. The cause for infection was thought to be poor nutritional status, nosocomial infection and patient's inability to afford costly antibiotics. In the series by Bhandari et al. ${ }^{[2]}$ Illizarov ring fixator is a good modality of treatment but is cumbersome for the patient and difficult to master by the surgeon as compared to LRS. According to a study done by Ajmera et al., LRS proved to be an effective modality of treatment in cases of open fractures tibia with bone loss as definite modality of treatment for damage control as well as for achieving union and lengthening ${ }^{[11]}$.

LRS fixator provides immediate stability to fracture fragments and allows immediate weight bearing which ultimately promotes early fracture healing and reduced financial burden.

\section{Conclusion}

Early stabilization of open tibial fracture with LRS with immediate soft tissue coverage resulted in good fracture union and minimal rates of complications compared to other modalities of treatment. It is cost effective with minimal hospital stay and early return to work. Over all morbidity is reduced and better patient satisfaction noted. LRS external fixation with the complexity of frame configuration provide 
good fixation. Resection of devitalized bone, simultaneous compression of the fracture gap or site and secondary limb lengthening by proximal corticotomy and compression and distraction techniques can be accompanied with the LRS external fixator with low rates of infection and non-union. Complications are minimal, with good range of movements at knee and ankle. It is not cumbersome like Ilizarov and easily maintainable ex-fixator. LRS external fixation technique is often successful in salvaging limbs which otherwise would have been at high risk for amputation.

\section{References}

1. Whittle AP. Wood II GW. Fractures of lower extremity. Canale ST. Campbell's operative orthopaedics. 10th ed. Philadelphia. 2003;2:754-73.

2. Behrens F. External fixation. $3^{\text {rd }}$ ed. In: Manual of internal fixation, Muller ME, Allgower M, Schneider R, Willinegger H, eds. Berlin: Springer-Verlag; 1999,36778.

3. Tucker HL, Kendra JC, Kinnebrew TE. Management of unstable open and closed tibial fractures using the Ilizarov method. Clinical orthopaedics and related research. 1992:1(280):125-35.

4. Bone LA, Stegemann PH, McNamara KE, Seibel RO. External fixation of severely comminuted and open tibial pilon fractures. Clinical orthopaedics and related research. 1993;1(292):101-7.

5. Tornetta P, Bergman M, Watnik N. Treatment of grade IIIB open tibial fractures. J Bone Joint Surg 1994;76$\mathrm{B}: 13$.

6. Hessmann M, Mattens M, Rumbaut J. Use of the unilateral external fixator (monofixator) in fracture treatment: experience in 50 fractures. Der Unfallchirurg. 1994;97(10):511-7.

7. Shaw DL, Lawton JO. External fixation for tibial fractures: clinical results and cost effectiveness. Journal of the Royal College of Surgeons of Edinburgh. 1995;40(5):344-6.

8. Checketts RG, Moran CG, Jennings AG. 134 tibial shaft fractures managed with the Dynamic Axial Fixator. Acta Orthopaedica Scandinavica. 1995;66(3):271-4.

9. Tu YK, Lin CH, Su JI, Hsu DT, Chen RJ. Unreamed interlocking nail versus external fixator for open type III tibia fractures. Journal of Trauma and Acute Care Surgery. 1995;39(2):361-7.

10. Edwards CC, Simmons SC, Browner BD, Weigel MC. Severe open tibial fractures. Results treating 202 injuries with external fixation. Clinical orthopaedics and related research. 1988;1(230):98-115.

11. Helland P, Bøe A, Mølster AO, Solheim E, Hordvik M. Open tibial fractures treated with the Ex-fi-re external fixation system. Clinical Orthopaedics and Related Research®. 1996;326:209-20. 